\title{
Calculated Late Time Spectra of Supernovae
}

\author{
Timothy S. Axelrod \\ Lawrence Livermore National Laboratory \\ Livermore, California \\ U.S.A. \\ October, 1987
}

\section{Introduction}

We consider here the nebular phase spectra of supernovae whose late time luminosity is provided by the radioactive decay of ${ }^{56} \mathrm{Ni}$ and ${ }^{56} \mathrm{Co}$ synthesized in the explosion, A broad variety of supernovae are known or suspected to fall in this category. This includes all SNIa and SNIb, and at least some SNII, in particular SN1987a. At sufficiently late times the expanding supernova becomes basically nebular in character due to its decreasing optical depth. The spectra produced during this stage contain information on the density and abundance structure of the entire supernova, as opposed to spectra near maximum light which are affected only by the outermost layers. A numerical model for nebular spectrum formation is therefore potentially very valuable for answering currently outstanding questions about the post-explosion supernova structure. As an example, we can hope to determine the degree of mixing which occurs between the layers of the "onion-skin" abundance structure predicted by current one dimensional explosion calculations.

In the sections which follow, such a numerical model is briefly described and then applied to SN1972e, a typical SNIa, SN1985f, an SNIb, and finally to SN1987a. In the case of SN1987a predicted spectra are presented for the wavelength range from 1 to 100 microns at a time 300 days after explosion.

\section{Numerical Model}

\subsection{Optical Depths}

As a rough approximation, an expanding supernova can be treated as nebular when the continuum optical depth to the center falls below unity. This occurs when

$$
t>228 \frac{\sqrt{\mathrm{KM} M}}{u_{9}}
$$

where $t$ is the time after explosion in days, $\boldsymbol{\kappa}$ is the average opacity in $\mathrm{cm}^{2} / \mathrm{gm}, M$ is the mass in solar masses, and $u_{9}$ is the expansion velocity in units of $10^{9} \mathrm{~cm} / \mathrm{sec}$. As discussed by a number of authors, for example [1], the average opacity is quite uncertain due to the effects of dense thickets of overlapping lines that occur in rapidly expanding material with substantial amounts of heavy elements. A lower limit is obtained by considering only electron scattering, so that

$$
\kappa \approx 0.4 \frac{\bar{Z}}{\bar{A}}
$$

where $\bar{Z}$ is the average degree of ionization and $\bar{A}$ is the average atomic weight. Light curve studies, for example [2], show this value for kappa is probably not grossly in error.

As will be described in the sections that follow, all the supernova models we consider consist of one or more regions containing heavy elements, possibly surrounded by outer regions of helium and hydrogen. Consider, for example, the model for SN1987a discussed in Section 6. At times near 300 days, the inner "metal"' region is roughly characterized by $\bar{Z} \approx .3, \bar{A} \approx 16, M \approx 2, u_{9} \approx 0.1$. The outer "helium" region has $\bar{Z} \approx 0.1, \bar{A} \approx 4, M \approx 12, u_{9} \approx 2$. If these $\bar{Z}$ values were constant in time, then equation 1 implies that the outer region is optically thin for $t>40$, while the inner metal region becomes thin only for $t>300 d$. So for this model, the nebular approximation is (at least possibly) reasonable for times of 300 days and later. As the above reasoning makes clear, at these times what optical depth there is comes dominantly from the inner metal region, with the outer helium region forming a very thin atmosphere above it. The situation for the other model classes is similar, but they become optically thin at much earlier times, due to their lower mass and higher expansion velocity. 


\subsection{Energy Deposition}

The power source for the optical spectrum is the radioactive decay of ${ }^{56} \mathrm{Ni}$ and ${ }^{56} \mathrm{Co}$, whose role in supernovae has been extensively discussed for nearly 20 years [3]. At the times under discussion here, the $\mathrm{Ni}$ has all decayed and the only significant energy production is due to Co. The numerical model operates by first calculating the energy deposition from the Co decay products throughout the nebula. The gamma deposition is determined by solving a 4 group steady state transfer equation in spherical geometry. The complex Co gamma emission spectrum is approximated by emission at a single characteristic energy, usually taken to be $1.5 \mathrm{mev}$, and these photons then are scattered into lower energy groups. At a scattering event the incident photon deposits a fixed fraction of its energy (taken from the data of Plechaty et al [4]) and then scatters isotropically at a correspondingly lower energy. Comparison of this approximation with a full Monte Carlo treatment of the gamma transport has shown that the accuracy achieved is more than adequate for the supernovae considered here.

In addition to the gammas, ${ }^{56} \mathrm{Co}$ emits a continuous positron spectrum accounting for $4 \%$ of the emitted energy on average. The positrons are expected to deposit nearly locally due both to their high energy deposition crossection and the large effect of even interstellar level magnetic fields on their effective path length. To allow a crude investigation of the effects of nonlocal deposition, the positrons are treated identically to the gammas, but with only a single group and with an energy deposition fraction which is always unity. The effective crossection can then be varied to simulate nonlocal deposition, with a large value resulting in local deposition.

\subsection{Temperature and Ionization State}

Energy deposition from ${ }^{56} \mathrm{Co}$ decay products causes both ionization and heating throughout the nebula. The ionization state and temperature at each point is determined by iteratively solving for a steady state in which ionization by fast particles and reabsorbed recombination radiation is balanced by radiative and dielectronic recombination; and in which heating is balanced by radiative cooling, principally from collisionally excited forbidden lines. This latter calculation is made more rapid by the use of cooling tables precalculated for each ion as a function of temperature and electron density. For the most part spatial coupling between different regions of the nebula is weak. The one important exception is that recombination radiation emitted in one region may transport to another and strongly affect the ionization balance there by causing increased ionization. This is treated by solving a full transfer equation for the recombination radiation and coupling it to the ionization balance equation.

\subsection{Spectrum Calculation}

Once the temperature and ionization state has been determined as a function of radius throughout the nebula, calculation of the emergent optical spectrum is straightforward. For each zone in the nebula, level populations for each ion are calculated by solving steady state rate equations. Then, for each radiative transition, the contribution to the emergent flux is calculated by using the line profile appropriate for an optically thin shell moving at the given velocity.

The numerical model is set up to handle atomic data for an arbitrary number of elements, and for each of these an arbitrary number of ionization stages can be incorporated. The atomic model for each ion in general consists of two separate models used for different purposes. The "x-ray" model is utilized for computing radiative and dielectronic recombination rates and photon generation from the cascade which follows such events. It is also used for the total photoionization crossections required to calculate the transport of the cascade radiation and its effects on the ionization balance. The "optical" model, on the other hand, is used for calculation of cooling rates and for generating the optical spectrum. In general, the "optical" model contains detailed information on low lying levels and the collisional and radiative transitions that connect them, while the " $\mathrm{x}$-ray" model contains a less detailed level structure that covers a much greater energy range.

The x-ray models used have been generated by a Hartree-Slater code $[5,6]$ of J. Scofield's. Radiative recombination rates are calculated from the photoionization crossections. Information for the optical models has been drawn from a wide variety of sources. The ions included in the present calculation are: FeI, II, III, IV, V, VI; CoI, II, III, IV, V; OI, II, III, IV; and HeI, II. Many important ions are not included, particularly in the intermediate $\mathrm{Z}$ range near Si. To minimize (but not eliminate) the effects of these 
omissions on the temperature and ionization structure, the missing ions are mapped to "glopium", a mythical element that cools and ionizes like $\mathrm{O}$, but produces no actual optical spectrum.

\section{Supernova Models}

The supernova models utilized below have all been generated by S. Woosley using the Kepler [7] supernova code. The evolution is followed with Kepler until the expansion becomes essentially homologous. At this point the Kepler information is transferred to the spectrum code. It is important to emphasize that once the supernova model has been selected the predicted spectra are generated without adjustable parameters. The adjustable parameters of the model are on the whole those with direct astrophysical relevance, for example the initial stellar mass and amount of mass loss. It is,.of course, feasible and useful to use the comparison of the observed and predicted spectra to refine the supernova model. This, however, has not yet been done except in a rough way. At present, the nebular spectra have been used only to choose broad classes of feasible models and rule out others. For example, the He detonation models for SNIa have been ruled out on the basis of the spectrum[8]. The results presented, therefore, must be viewed accordingly as generic to a whole class of models whose details are still unresolved.

\section{Application to SNIa}

The model chosen for an SNIa is a carbon deflagration in a white dwarf of $1.4 \mathrm{M}_{\odot}$ [9]. The "flame speed", which must currently be chosen somewhat arbitrarily, has been given an intermediate value. The explosion produces $1.0 \times 10^{51}$ ergs and $0.51 \mathrm{M}_{\odot}$ of ${ }^{56} \mathrm{Ni}$. The optical spectrum from the numerical model is compared to the observation of SN1972e at a time 264 days after explosion[10]. The spectrum is dominated by [FeII] and [FeIII]. The feature near 6000 Angstroms has been previously identified with [CoIII] and shown to decay in a manner consistent with the radioactive decay of ${ }^{56} \mathrm{Co}[11]$.

\section{Application to SNIb}

SNIb have only recently been recognized as a distinct class of supernovae [12]. At present there are only two SNIb for which observed spectra are available during the nebular phase. These are SN1983n [13] and SN1985f $[14,15]$. The progenitors of these events are still uncertain. Here we consider as a possibility a Wolf-Rayet star of $4 \mathrm{M}_{\odot}$ that has evolved from a $15 \mathrm{M}_{\odot}$ main sequence star. The star was evolved with Kepler to the point of Fe core collapse. The dynamics of the core collapse and the subsequent explosion have not been followed for this model, but rather simulated with a mass cut and a piston that transfers $2.5 \times 10^{51}$ ergs to the remaining star. $0.06 \mathrm{M}_{\odot}$ of ${ }^{56} \mathrm{Ni}$ are synthesized and ejected in the resulting explosion. The optical spectrum from this model at a time 200 days after explosion are compared with the spectrum of SN1985f as observed in April, 1985 [15]. The time of explosion of SN1985f is not well known, so this choice of times is in itself speculative. The two spectra have more than a superficial resemblance, however. The dominant features in the calculated spectrum are due to OI and OII, and are matched well in intensity in the observation, although the widths are too great and the lineshape too flat-topped. The observed spectrum has a continuum which is absent in the calculation. This continuum probably results from the large number of allowed lines present in the Fe region, untreated in this model, which in the presence of large velocity gradients can generate a "quasi-continuum" across the entire optical wavelength region [11].

If SN1985f was indeed 200 days old at the time of the observed spectrum, the model spectrum can be used to infer a distance to $\mathrm{M} 83$. This distance is $7 \mathrm{Mpc}$, considered within the present uncertainties in the distance to this galaxy [13]. It is therefore worth noting that the model generates both a spectrum and a luminosity in reasonable agreement with observations in spite of the fact that it has only $0.6 \mathrm{M}_{\odot}$ of $O$ compared with the $5 \mathrm{M}_{\odot}$ that has been previously claimed to be a requirement [16].

\section{Application to SN1987a}

Models for SN1987a have recently been extensively discussed by Woosley [17]. We have used model 10H from that paper for the spectrum calculation. As discussed there in detail, this model is the core of a $20 \mathrm{M}_{\odot}$ star which has lost $4 \mathrm{M}_{\odot}$ of mass in a wind. The core collapse and subsequent explosion produces ejecta with $1.4 \times 10^{51} \mathrm{ergs}$ and $0.08 \mathrm{M}_{\odot}$ of ${ }^{56} \mathrm{Ni}$. As discussed in an earlier section of this paper, this model is not thin in the continuum until roughly 300 days after explosion. In fact, the situation is worse than this, since 
the optical region remains obscured by allowed lines (principally from $\mathrm{Fe}$ ) until considerably later times. In the infrared, however, this model is predicted to be quite transparent at this time, and we therefore present the predicted spectrum at 300 days. The major caveat is that for this model the neglect of allowed lines may result in considerable errors in the calculated temperature and ionization state. Nontheless, it is clear that the infrared spectrum should contain a wealth of information on the abundance, temperature, and ionization state of the ejecta. The late time spectrum of this model has also been calculated and discussed by Fransson and Chevalier[18]

\section{Acknowledgements}

I am grateful to Claess Fransson for making many valuable points on the late time spectra of supernovae. This work performed under the auspices of the U. S. Department of Energy by the Lawrence Livermore National Laboratory under contract No. W-7405-ENG-48.

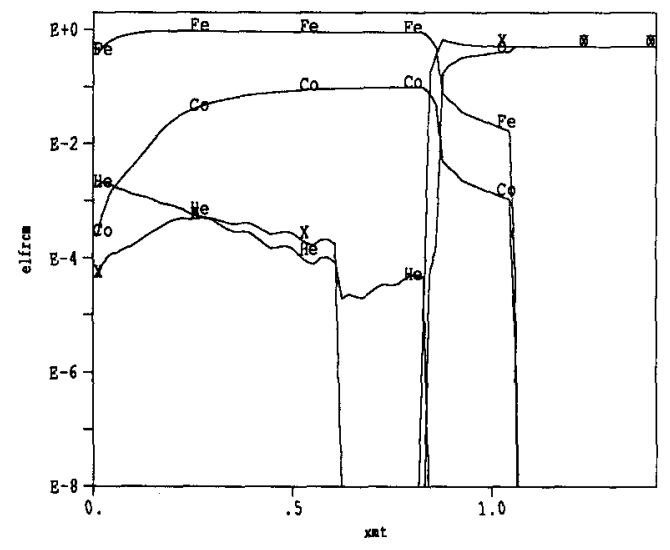

Fig. 1a

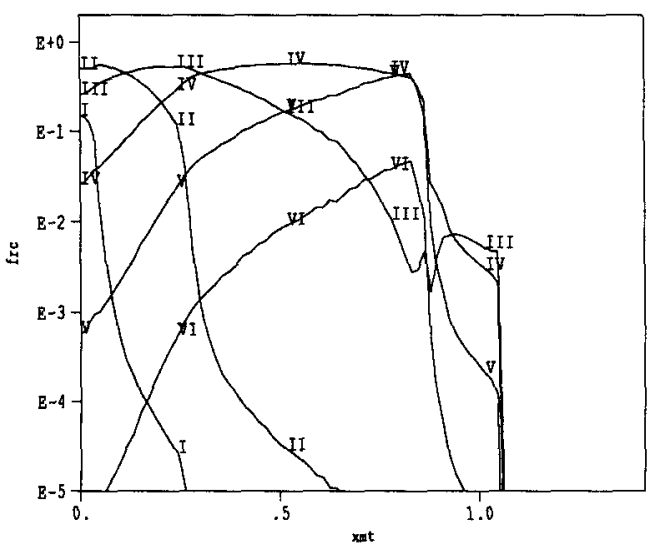

Fig. 1c

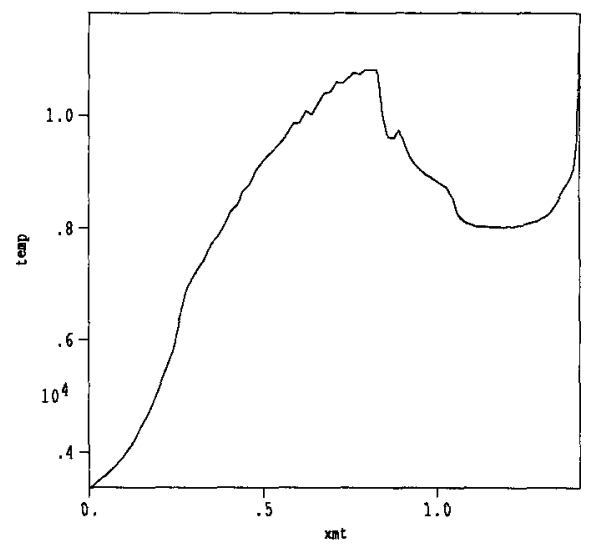

Fig. $1 b$

0

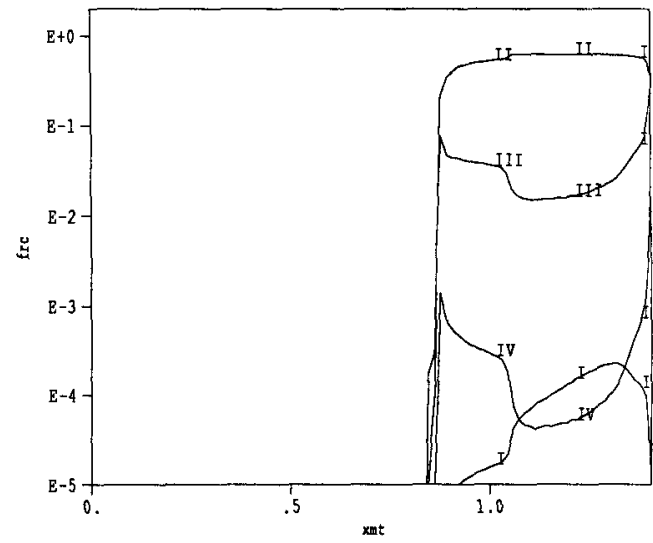

Fig. 1d

1. Element mass fractions (Fig. 1a), temperature in degrees $\mathrm{K}$ (Fig. 1b), ionization state of Fe (Fig. 1c) and $O$ (Fig. 1d) are shown for the SNIa model discussed in Section 4. The $x$ axis is the mass coordinate in $\mathrm{M}_{\odot}$ 


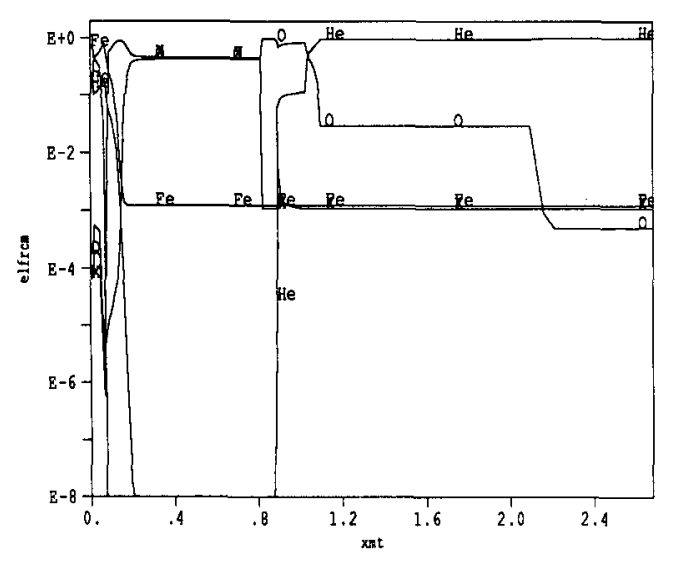

Fig. $2 a$

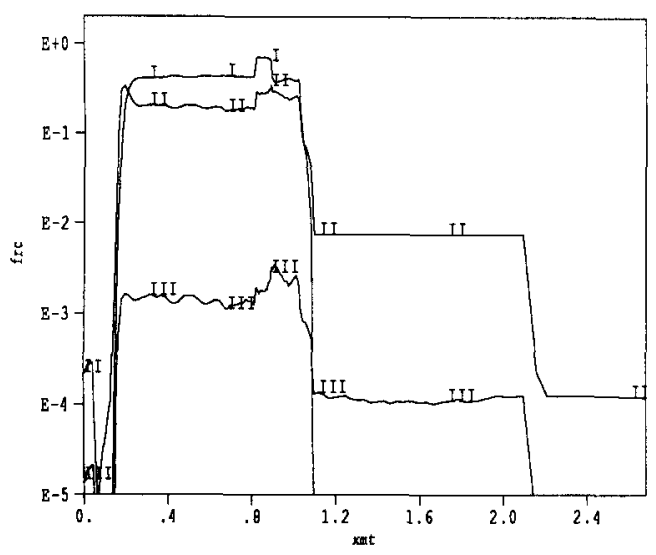

Fig. 2C

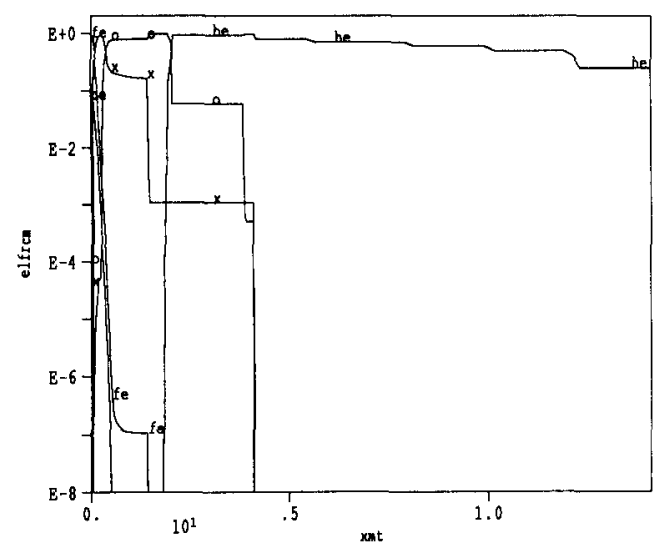

Fig. $3 a$

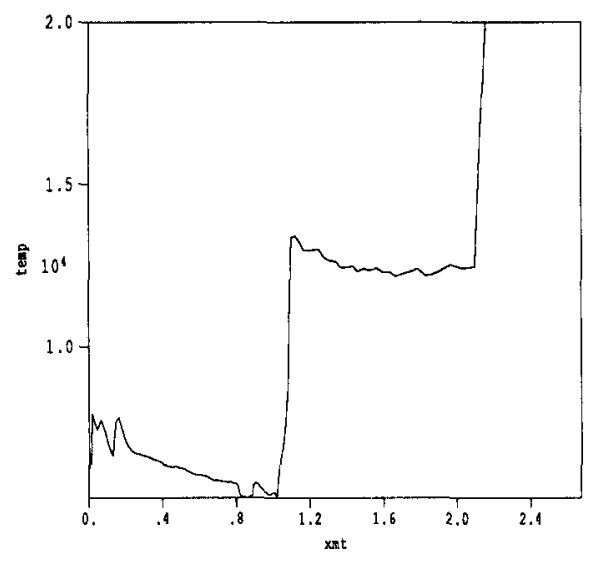

Fig. 2b

2. Same as Fig. 1, but with Fe ionization state omitted, and for the SNIb model of Section 5.

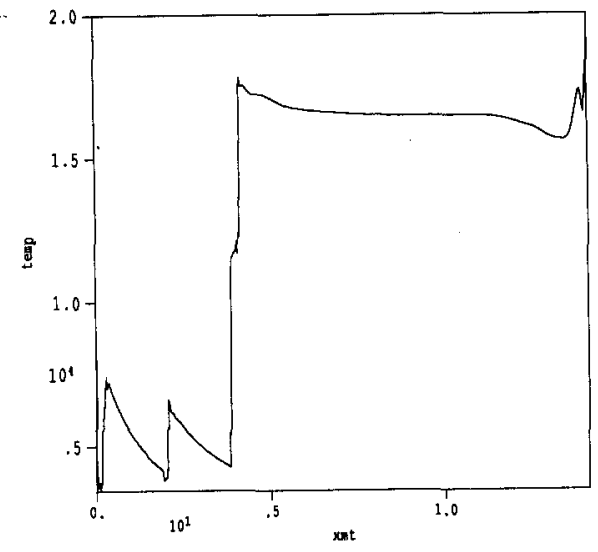

Fig. 3b 


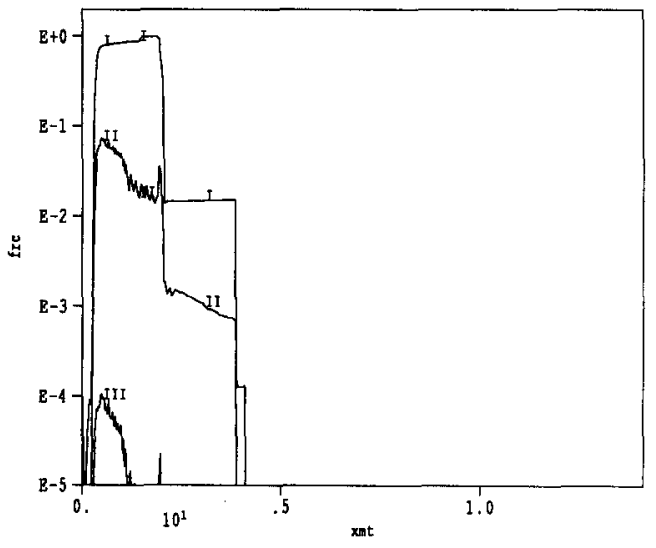

Fig. 3c
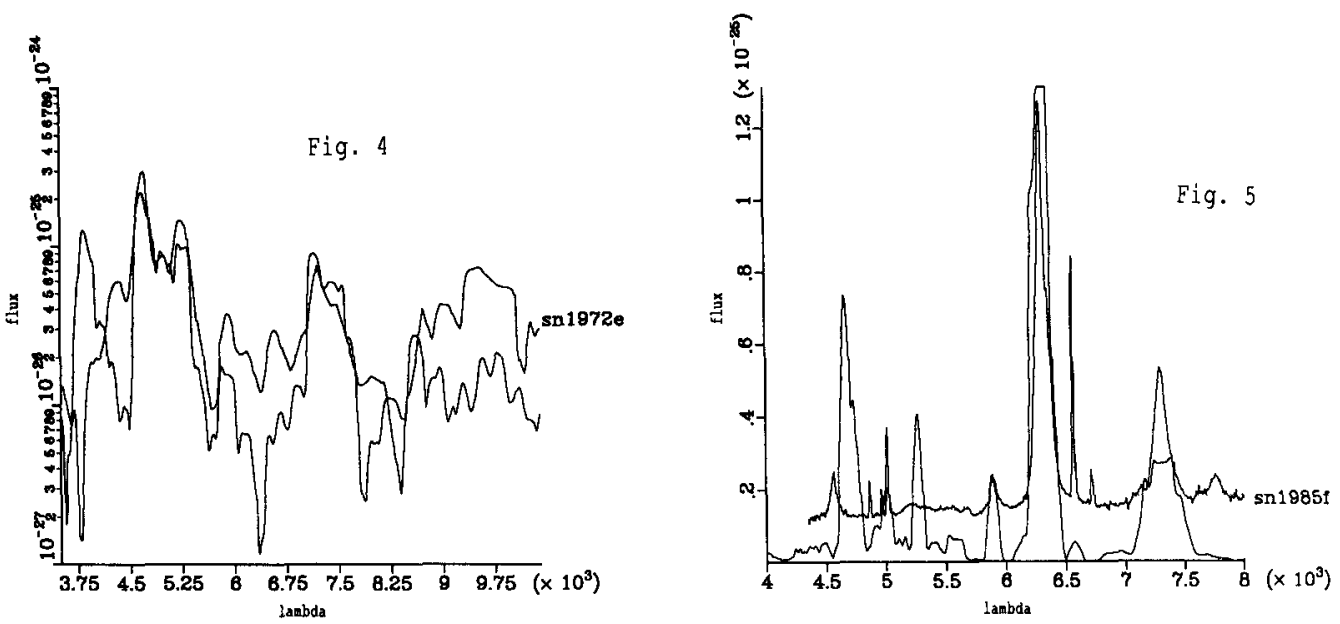

4. Comparison of the SNIa model spectrum with that of SN1972e 264 days after explosion. The flux units are $\mathrm{erg} / \mathrm{sec} / \mathrm{cm}^{2} / \mathrm{Hz}$. The wavelength is in Angstroms.

5. Comparison of the SNIb model spectrum 200 days after explosion with that of SN1985f in April 1985 . 


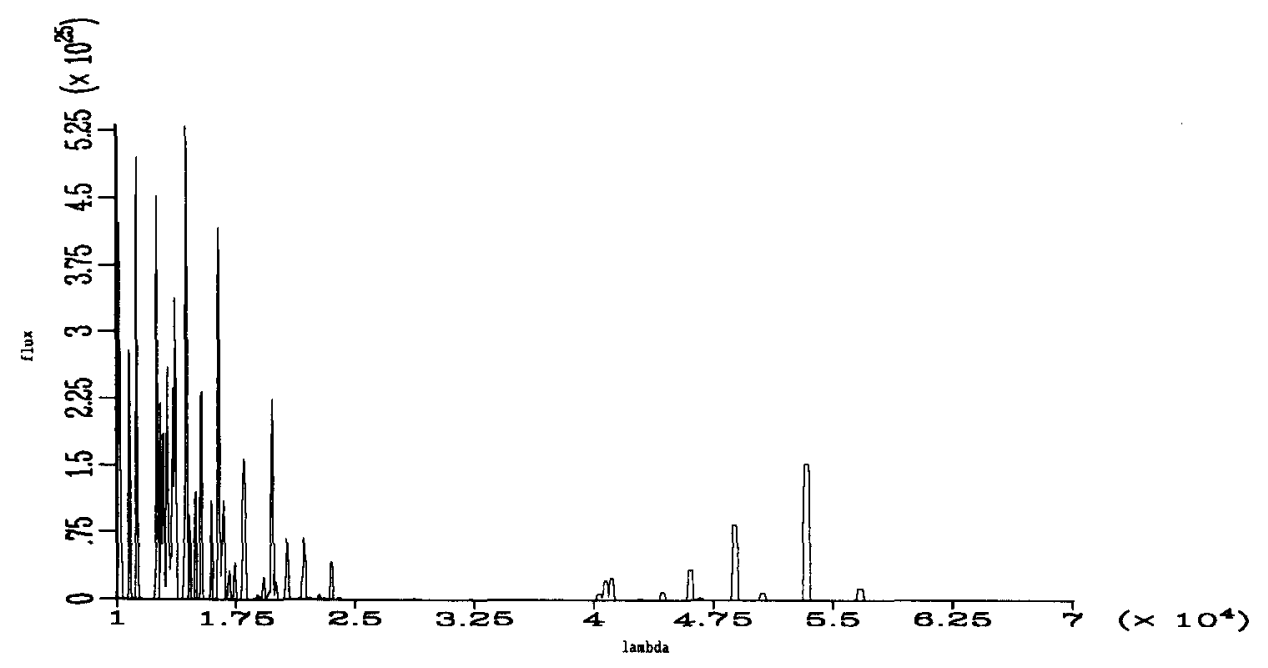

Fig. $6 a$

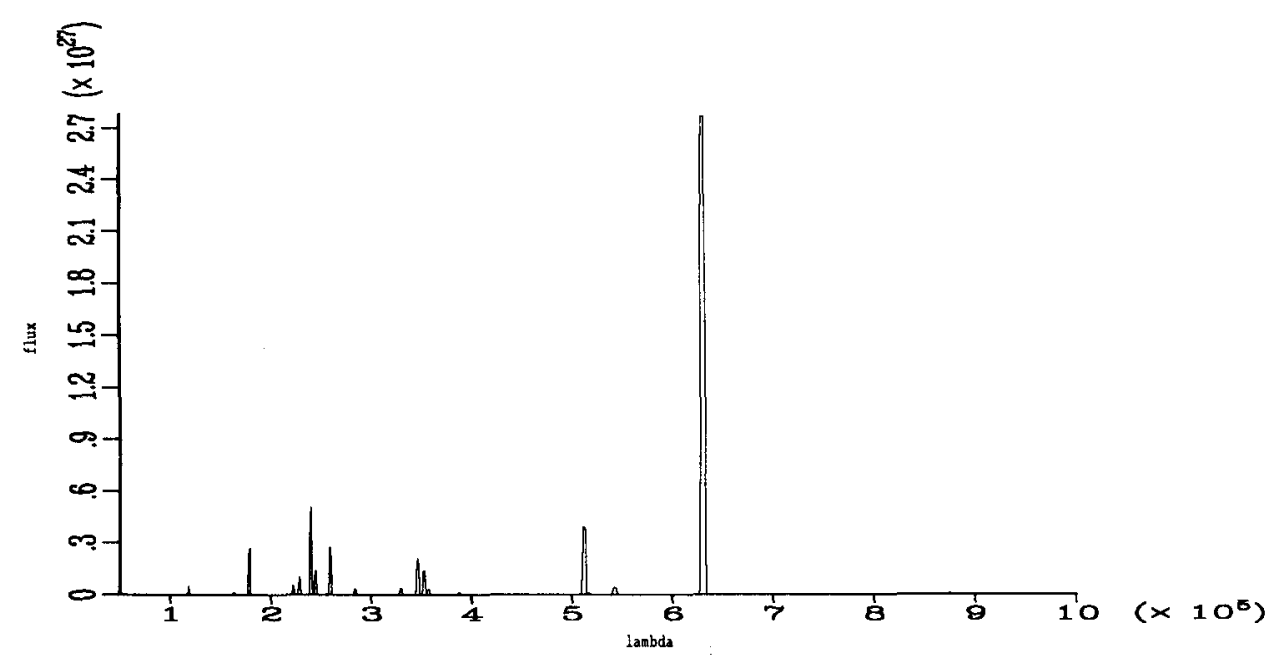

Fig. $6 \mathrm{~b}$

6. Predicted IR spectrum of the SN1987a model at a time 300 days after explosion. The flux units are $\mathrm{erg} / \mathrm{sec} / \mathrm{Hz}$.

\section{References}

1. A. H. Karp, K. L. Chan, G. J. Lasher, and E. E. Salpeter, Ap. J., vol. 214, p. 161, 1977.

2. W. D. Amett, Ap. J., vol. 253, p. 785, 1982.

3. S. A. Colgate and C. McKee, Ap. J., vol. 157, p. 623, 1969.

4. E. F. Plechaty, D. E. Cuilen, and R. J. Howerton, Lawrence Livermore National Laboratory UCRL-50400, 1981.

5. J. H. Scofield, Phys. Rev., vol. 179, p. 9, 1969.

6. J. H. Scofield, Lawrence Livermore National Laboratory UCRL-51231, 1972. 
7. T. A. Weaver, G. B. Zimmerman, and S. E. Woosley, Ap.J., vol. 225, p. 1021, 1978.

8. S. E. Woosley, T. S. Axelrod, and T. A. Weaver, in Stellar Nucleosynthesis, ed. C. Chiosi, p. $263,1984$.

9. S. E. Woosley and T. A. Weaver, in Radiation Hydrodynamics in Stars and Compact Objects, ed. D. Mihalas, p. 91, 1986.

10. R. P. Kirshner and J. B. Oke, Ap. J., vol. 200, p. 574, 1975.

11. Axelrod, T. S., Lawrence Livermore National Laboratory UCRL-52994, 1980.

12. J. C. Wheeler and R. Levreault, Ap. J., vol. 219, p. L17, 1985.

13. C. M. Gaskell, E. Cappellaro, H. L. Dinerstein, D. R. Garnett, R. P. Harkness, and J. C. Whecler, Ap. J., vol. 306, p. L77, 1986.

14. A. V. Filippenko and W. L. W. Sargent, Nature, vol. 316, p. 407, 1985.

15. A. V. Filippenko and W. L. W. Sargent, Astron. J., vol. 91, p. 691, 1986.

16. M. C. Begelman and C. L. Sarazin, Ap. J., vol. 302, p. L59, 1986.

17. S. E. Woosley, Submitted to Ap. J., 1987.

18. C. Fransson and R. A. Chevalier, Preprint, 1987. 